\title{
Exercise Barriers in Korean Colorectal Cancer Patients
}

\author{
Dong-Woo Kang ${ }^{1}$, Jae Youn Chung ${ }^{1}$, Mi Kyung Lee ${ }^{1}$, Junga Lee ${ }^{1}$, Ji-Hye Park ${ }^{1}$, \\ Dong-il Kim ${ }^{1}$, Lee W Jones ${ }^{2}$, Joong Bae Ahn ${ }^{3}$, Nam Kyu Kim ${ }^{4}$, Justin Y Jeon ${ }^{1 *}$
}

\begin{abstract}
Background: It has been proved that participating in exercise improves colorectal cancer patients' prognosis. This study is to identify barriers to exercise in Korean colorectal cancer patients and survivors. Materials and Methods: A total of 427 colorectal cancer patients and survivors from different stages and medical status completed a self-administered questionnaire that surveyed their barriers to exercise and exercise participation. Results: The greatest perceived exercise barriers for the sampled population as a whole were fatigue, low level of physical fitness, and poor health. Those under 60 -years old reported lack of time $(p=0.008)$, whereas those over 60 reported low level of physical fitness $(p=0.014)$ as greater exercise barriers than their counterparts. Women reported fatigue as a greater barrier than men $(p<0.001)$. Those who were receiving treatment rated poor health $(p=0.0005)$ and cancer-related factors as greater exercise barriers compared to those who were not receiving treatment. A multivariate model found that other demographic and medical status were not potential factors that may affect exercise participation. Further, for those who were not participating in physical activity, tendency to be physically inactive $(p<0.001)$ and lack of exercise skill $(p<0.001)$ were highly significant barriers, compared to those who were participating in physical activity. Also, for those who were not meeting ACSM guidelines, cancer-related exercise barriers were additionally reported $(\mathbf{p}<0.001)$, compared to those who were. Conclusions: Our study suggests that fatigue, low level of physical fitness, and poor health are most reported exercise barriers for Korean colorectal cancer survivors and there are differences in exercise barriers by age, sex, treatment status, and physical activity level. Therefore, support for cancer patients should be provided considering these variables to increase exercise participation.
\end{abstract}

Keywords: Exercise - barrier - colorectal cancer - physical activity

Asian Pac J Cancer Prev, 15 (18), 7539-7545

\section{Introduction}

The third most diagnosed cancer in men and women in US is colorectal cancer with approximately 142,820 estimated incidence and 50,830 deaths in 2013 (Siegel et al., 2013). Despite the large number of people diagnosed with colorectal cancer, the mortality rate for this disease has decreased in recent years due to improvements in treatment and early detection (Jemal et al., 2011). In Korea, colorectal cancer incidence rates in both genders also increased $5.9 \%$ annually over the last decade, while 5 -year survival rates improved up to almost $73 \%$ with $17.8 \%$ of annual increase between 2006 and 2010 (Jung et al., 2013). Therefore, the number of colorectal cancer survivors is expected to increase continuously. This trend in colorectal cancer incidence and mortality implies that managing physical health and quality of life throughout the cancer continuum is important. Recent meta-analysis showed that the level of physical activity participation either before or after the diagnosis of colorectal cancer was negatively associated with reduced the colorectal specific and all-cause mortality (Je et al., 2013). Also, a recent article concluded through previous literatures that the effect of physical activity on the cancer risk is particularly stronger for colon cancer than other types of cancers (Kruk and Czerniak, 2013). In this respect, many studies have showed positive impact of exercise and physical activity participation on quality of life and other psychological health of cancer survivors (Cadmus et al., 2009; Courneya et al., 2003; Maryam et al., 2010; Peddle et al., 2008; Shariati et al., 2010; Sherman et al., 2010). In addition, exercise positively improves physical function and biomarkers related to colorectal cancer, such as insulin resistance, insulin-like growth factor (IGF)1 , tumor necrosis factor (TNF)-alpha, and adiponectin (Cheville et al., 2013; Lee et al., 2013). It has also been found that exercise shortens the length of hospitalization and time to flatus even for inpatients who just underwent colorectal cancer surgery, which may improve long-term colorectal cancer prognosis (Ahn et al., 2013).

${ }^{1}$ Department of Sport and Leisure Studies, Yonsei University, ${ }^{3}$ Department of Internal Medicine, ${ }^{4}$ Department of Surgery, Yonsei University College of Medicine, Seoul, Korea, ${ }^{2}$ Memorial Sloan Kettering Cancer Center, Newyork, USA *For correspondence: jjeon@yonsei.ac.kr,NAMKYUK@yuhs.ac 
Although the positive impacts of exercise and physical activity on colorectal cancer have been acknowledged, many colorectal cancer patients still do not participate in exercise. The American College of Sport Medicine (ACSM) provides physical activity guidelines for the general population (150 minutes of moderate to vigorous physical activity per week) to clinically improve physical health, and it is also applicable to cancer survivors when the individual survivors' health status are considered (Schmitz et al., 2010). However, Chung et al. recently reported that only $7.6 \%$ and $25.2 \%$ of colorectal cancer patients were meeting ACSM physical activity guidelines during and after treatment, respectively (Chung et al., 2013). Likewise, Bellizzi et al. showed that only $23.7 \%$ of colorectal cancer patients met ACSM physical activity guidelines after diagnosis, which was less than the physical activity participation rates for other types of cancer patients (Bellizzi et al., 2005).

Several studies have been conducted to explore the barriers that keep cancer patients from participating in exercise, including breast (Nelson, 1991; Leddy, 1997; Rogers et al., 2006; Rogers et al., 2007; Courneya et al., 2008; Gho et al., 2010; Loh et al., 2012; Ottenbacher et al., 2013), prostate (Ottenbacher et al., 2013), and head and neck (Rogers et al., 2008) cancer. For colorectal cancer, Courneya et al. (2005) identified exercise barriers during an exercise program intervention and Lynch et al. (2010) reported barriers to physical activity for colorectal cancer patients according to the time period after diagnosis. However, to our knowledge, there were no studies on barriers to regular exercise participation in a typical day setting among colorectal cancer patients. In addition, although perceived impediments to exercise participation vary according to cancer type, modality, treatment type, and psychological and social context, each showing unique and different biological and psychosocial attributes (Brawley et al., 2003), none of previous studies considered the participants' demographics, treatment status, and the level of physical activity participation. Therefore, the purpose of the current study is to identify barriers to exercise in colorectal cancer patients according to their demographic profile, treatment status, and physical activity level.

\section{Materials and Methods}

\section{Study design and participant}

The overall study method was reported elsewhere (Chung et al., 2013). In brief, this cross-sectional study was designed to understand exercise barriers perceived by colorectal cancer patients. Patients who were diagnosed with stage I-III colorectal cancer were eligible, and the survey was conducted from July 2010 to August 2011 in Shinchon Severance Hospital in Korea. We were provided with the hospital patient registry and medical records from the Colorectal Cancer Clinic. Study explanation and consent form were provided to eligible patients, and those who signed the consent form were then asked to answer the questionnaire about exercise barriers. Welltrained research staffs helped the participants answer the questionnaire by interviewing face-to-face. This study
Table 1. Demographic and Medical Characteristics $(\mathrm{n}=427)$

\begin{tabular}{|c|c|c|c|}
\hline Variable & Categories & $\mathrm{n}$ & $\%$ \\
\hline \multicolumn{4}{|l|}{ Demographics } \\
\hline \multirow[t]{2}{*}{ Sex } & Male & 269 & 63 \\
\hline & Female & 158 & 37 \\
\hline \multirow[t]{2}{*}{ Age } & $<60$ & 201 & 47.1 \\
\hline & $\geq 60$ & 199 & 46.6 \\
\hline \multirow[t]{4}{*}{ Education } & Secondary school & 98 & 23 \\
\hline & High school & 165 & 38.6 \\
\hline & College & 101 & 23.7 \\
\hline & Post-graduate & 60 & 14.1 \\
\hline \multicolumn{4}{|c|}{ Household income (per month) } \\
\hline & No & 108 & 25.3 \\
\hline & $\leq \$ 1,000$ & 33 & 7.7 \\
\hline & $\$ 1,001-\$ 2,000$ & 47 & 11 \\
\hline & $\$ 2,001-\$ 3,000$ & 60 & 14.1 \\
\hline & $\$ 3,001-\$ 4,000$ & 52 & 12.2 \\
\hline & $\$ 4,001-\$ 5,000$ & 38 & 8.9 \\
\hline & $\geq \$ 5,001$ & 74 & 17.3 \\
\hline \multirow[t]{4}{*}{ Marital status } & Married & 375 & 87.8 \\
\hline & Single & 13 & 3 \\
\hline & Bereavement & 31 & 7.3 \\
\hline & Divorce & 5 & 1.2 \\
\hline \multicolumn{4}{|l|}{ Medical history } \\
\hline \multirow[t]{9}{*}{ Tumor location } & Colon & 10 & 2.3 \\
\hline & Ascending colon & 33 & 7.7 \\
\hline & Transverse colon & 13 & 3 \\
\hline & Descending colon & 17 & 4 \\
\hline & Rectrosigmoid colon & 15 & 3.5 \\
\hline & Sigmoid colon & 71 & 16.6 \\
\hline & Rectal & 204 & 47.8 \\
\hline & Cecal, cecum & 5 & 1.2 \\
\hline & Anal & 1 & 0.2 \\
\hline \multirow[t]{2}{*}{ Treatment } & On-treatment & 132 & 30.9 \\
\hline & Off-treatment & 286 & 67 \\
\hline \multirow[t]{4}{*}{ Cancer stage } & I & 95 & 22.2 \\
\hline & II & 102 & 23.9 \\
\hline & III & 106 & 24.8 \\
\hline & IV & 18 & 4.2 \\
\hline \multirow[t]{2}{*}{ Surgery } & Yes & 380 & 89 \\
\hline & No & 43 & 10.1 \\
\hline \multirow[t]{2}{*}{ Ostomy } & Yes & 52 & 12.2 \\
\hline & No & 185 & 43.3 \\
\hline \multicolumn{4}{|c|}{ Physical activity level } \\
\hline \multicolumn{4}{|c|}{ Physical activity participation } \\
\hline & Yes ( $>0$ MET-hr/wk) & 315 & 73.8 \\
\hline & No (not at all) & 112 & 26.2 \\
\hline \multicolumn{4}{|c|}{ Meeting ACSM guidelines } \\
\hline & Yes & 84 & 19.7 \\
\hline & No & 343 & 80.3 \\
\hline
\end{tabular}

*Numbers of participants may not sum up 427 due to missing data. MET-hr/wk: Metabolic equivalent task-hour/week. ACSM guidelines: more than 150 minutes per week of moderate to vigorous physical activity

was approved by the Institutional Review Board at Yonsei University College of Medicine.

\section{Exercise barrier assessment}

The Exercise Barrier Questionnaire for Older Adults (Seong et al., 2008) was adopted and revised for in this study. The questionnaire was amended by adding cancerrelated barriers that were reported in previous studies (Courneya et al., 2008; Irwin, 2009), and then validated by experts review and panel discussions. The final revised 
questionnaire comprised 21 questions (See appendix 1), including cancer patient-specific questions on topics such as 'fear of adverse effects on cancer site or treatment' and 'pain at site of surgery during exercise'. Subjects were asked to answer each question using a 5-point Likert scale: Strongly Disagree (1 point), Disagree ( 2 points), Neutral (3 points), Agree (4 points), and Strongly Agree (5 points). The mean values of each question were then calculated, and higher mean value denotes greater exercise barrier for the participants.

\section{Statistical analysis}

Descriptive analyses were used for the participants' baseline characteristics. To analyze differences in exercise barriers between subgroups, subjects were divided according to demographics (sex, age, education, household income, and marital status), and medical history (tumor location, treatment, cancer stage, surgery, and ostomy). Also, the amount of physical activity participation (participating $v s$ not participating in physical activity, meeting $v s$ not meeting ACSM guidelines) was used for further analysis. Data are presented as mean and standard deviation (SD), or mean and standard error (SE). T-tests were used to analyze statistical differences in exercise barriers between two groups divided by demographics and medial history. To identify potential factors that may affect exercise participation, we analyzed using a multivariate model, and to analyze differences in exercise barriers according to treatment status and physical activity level, ANCOVA were used with adjusted age and gender. $\mathrm{p}<0.05$ was considered statistically significant.

\section{Results}

We completed a total of 431 surveys. After excluding four participants who responded to less than $50 \%$ of the questionnaire, data for 427 participants were analyzed for this study. The participants' demographics and medical profiles are presented in Table 1. In brief, $63.0 \%$ were male, $47.1 \%$ were over 60 years old, $37.8 \%$ were college-educated, $28.4 \%$ had a monthly household income of more

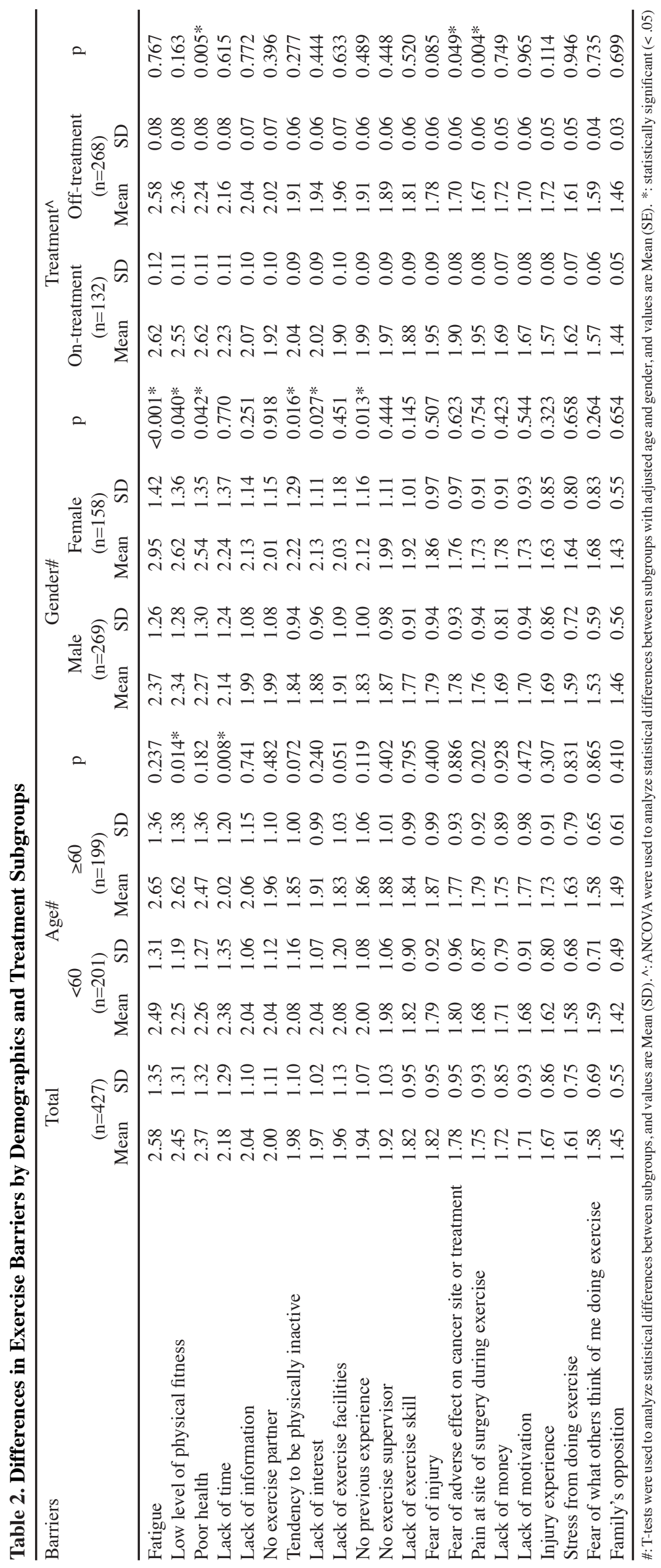

Asian Pacific Journal of Cancer Prevention, Vol 15, 2014 
than $\$ 3,000$, and $87.8 \%$ were married. In terms of medical status, $38.3 \%$ had colon cancer and $48.0 \%$ had rectal cancer, $30.9 \%$ were undergoing adjuvant or neoadjuvant treatment, including chemotherapy, radiation therapy, or both, $70.9 \%$ were cancer stage I-III $89 \%$ had undergone surgery, and $12.2 \%$ had an ostomy bag. For physical activity level, $73.8 \%$ were participating in physical activity, 26.2\% were sedentary. Those meeting ACSM guidelines were $19.7 \%$ of all participants, but were $26.7 \%$ of those who were participating in physical activity.

The barriers to exercise for colorectal cancer patients are described in Table 2. The barriers are sorted in descending order by mean values: A higher mean value indicates a greater barrier perceived by the participants. Among 21 exercise barriers in the questionnaire, fatigue was perceived as the greatest barrier to exercise in colorectal cancer patients, followed by low level of physical fitness, poor health, and lack of time. Basically, we analyzed the barriers to exercise in colorectal cancer patients by demographics. Subjects over 60 years of age answered that low level of physical fitness was their greater barrier than those under 60 years old $(\mathrm{p}=0.014)$, whereas subjects under 60 years old felt that lack of time was their greater barrier to exercise than those over 60 years old $(\mathrm{p}=0.008)$. Female patients considered fatigue $(\mathrm{p}<0.001)$, low level of physical fitness $(\mathrm{p}=0.040)$, poor health $(\mathrm{p}=0.042)$, tendency to be physically inactive $(\mathrm{p}=0.016)$, lack of interest $(\mathrm{p}=0.027)$, and no previous experience $(\mathrm{p}=0.013)$ significantly greater barriers than male patients. There were no significant difference in exercise barriers according to education (secondary and high school vs college and post-graduate), household income per month (bottom 50\% vs top 50\%), and marital status (married $v s$ either single, bereavement, or divorce) (not presented). We used a multivariate model to find potential factors that may influence whether patients participate in exercise or not. As a result, only treatment status was identified as a factor that affect exercise participation (not presented), and patients who completed treatment was participating in exercise more than those who were not completed treatment (odds ratio: $2.653,95 \%$ confidential interval: $1.181-5.958)$. When we divided the subjects according to treatment status, those currently undergoing chemotherapy, radiation therapy, or both treatments reported greater feelings of poor health $(p=0.005)$, and fear of adverse effect on cancer site or treatment $(\mathrm{p}=0.049)$, and pain at site of surgery during exercise $(\mathrm{p}=0.004)$ as barriers to exercise than those who were not undergoing treatment. Tumor location (colon $v s$ rectal), cancer stage (I-II vs III-IV), surgery (Yes $v s$ No), and ostomy (Yes $v s$ No) were not significant factors that could affect exercise barriers, as they did not show statistical differences between groups.

We further compared barriers between subgroups according to the level of physical activity participation (Table 3). Subjects who were not participating in physical activity at all indicated that fatigue $(\mathrm{p}=0.001)$, low level of physical fitness $(\mathrm{p}=0.006)$, poor health $(\mathrm{p}=0.005)$, lack of time $(\mathrm{p}=0.003)$, lack of information $(\mathrm{p}=0.033)$, tendency to be physically inactive $(\mathrm{p}<0.001)$, lack of interest $(\mathrm{p}=0.016)$, no previous experience $(\mathrm{p}=0.004)$, no exercise supervisor $(\mathrm{p}=0.030)$, lack of exercise skill $(\mathrm{p}<0.001)$, pain at site of surgery during exercise $(\mathrm{p}=0.049)$, lack of money $(\mathrm{p}=0.031)$, lack of motivation $(\mathrm{p}=0.001)$, stress from doing exercise $(\mathrm{p}=0.009)$, and fear of what others think of me doing exercise $(\mathrm{p}=0.043)$ were significantly greater barriers to exercise than those who were participating in physical activity. Among those who were participating

Table 3. Differences in Exercise Barriers according to Physical Activity Level with Age and Gender Control

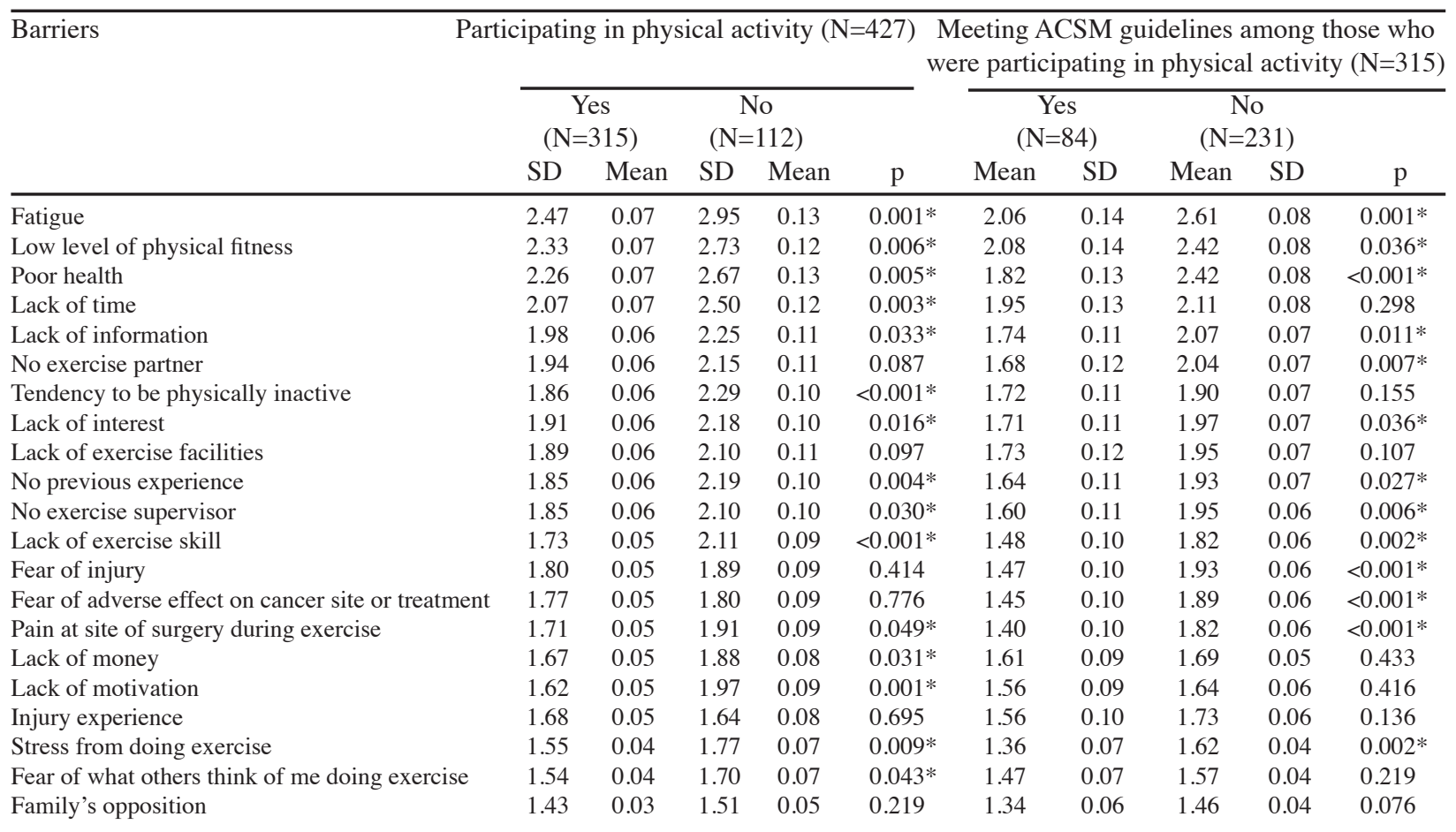

Values are Mean (SE). ANCOVA were used to analyze statistical differences between subgroups with adjusted age and gender. ACSM guidelines: more than 150 minutes per week of moderate to vigorous physical activity. $\wedge^{\wedge}: *$ : statistically significant $(<.05)$ 
in any amount of physical activity, patients who did not meet the ACSM physical activity guidelines found that fatigue $(p=0.001)$, low level of physical fitness $(p=0.036)$, poor health $(\mathrm{p}<0.001)$, lack of information $(\mathrm{p}=0.011)$, no exercise partner $(\mathrm{p}=0.007)$, lack of interest $(\mathrm{p}=0.036)$, no previous experience $(\mathrm{p}=0.027)$, no exercise supervisor $(p=0.006)$, lack of exercise skill $(p=0.002)$, fear of injury $(\mathrm{p}<0.001)$, fear of adverse effect on cancer site or treatment $(\mathrm{p}<0.001)$, pain at site of surgery during exercise $(p<0.001)$, and stress from doing exercise $(p=0.002)$ were significantly greater barriers to exercise than those who met the ACSM guidelines.

\section{Discussion}

Understanding exercise barriers in colorectal cancer patients is an essential step towards effectively increasing their exercise participation in order to improve physical and mental heaths. In our study, fatigue, low level of physical fitness, and poor health were highly ranked barriers to exercise, followed by lack of time and lack of information. The results of this study are in agreement with the results of previous studies in that fatigue was considered one of the major barriers to exercise in colorectal cancer patients (Courneya et al., 2005; Lynch et al., 2010); however, the most frequently reported exercise barrier among colorectal cancer patients in a previous study (Courneya et al., 2005) was 'lack of time/ too busy'. One reason for this discrepancy may be because Courneya et al. defined exercise barriers as excuses for not attending regular, supervised exercise sessions, whereas an exercise barrier in our study was defined as an exercise impediment that the subjects generally experienced in a typical day (Courneya et al., 2005). Lynch et al. also investigated barriers to physical activity among colorectal cancer patients and found that the most frequently reported barrier was 'already active enough' (Lynch et al., 2010); however, their questionnaire was different from ours in that they investigated barriers to 'physical activity' while we explored barriers to 'exercise', which are clearly different in that exercise requires more regular and structured physical movement with short- and long-term goals (Caspersen et al., 1985).

We examined differences in exercise barriers among demographic and medical status subgroups. Lack of time was a greater barrier for subjects under 60 years old, which may be because younger subjects are still working and have less time for exercise than their over-60 counterparts, while subjects over 60 years old reported low level of physical fitness as a greater barrier than those under 60 years old. Meanwhile, women reported fatigue as the greatest barrier with the highest mean value as high as 2.95 , which is the higher score than men. This may imply that women with colorectal cancer experience greater fatigue as an exercise barrier than men. On top of that, many women subjects had not participated in exercise for the reason that they are not physically active. Therefore, it appears to be important for women not only to relieve their fatigue and to increase physical fitness and health, but also to inform how much exercise is important for colorectal cancer patients and why they are recommended to participate in exercise. Subjects who were receiving cancer treatment were more likely to report fear of adverse effect on treatment and pain at surgery site, as well as poor health, as exercise barriers than those who were offtreatment. Although we expected that subjects receiving treatment would more likely cite fatigue as an exercise barrier because fatigue is a common complaint for cancer patients and it has been reported to increase when they are receiving treatment (Servaes et al., 2002; Spazzapan et al., 2004), this result suggests that the actual impediment to exercise for the subjects who are undergoing treatment may be poor health rather than fatigue, and there are also fear of adverse effect and pain from exercise. Therefore, it will be important for those who are receiving treatment to be educated on safety of exercise on cancer site, as well as to improve their physical fitness and health.

Furthermore, we analyzed differences in exercise barriers between those who were not participating in physical activity at all and those who were participating in any amount of physical activity. Since it has been found that colorectal cancer patients who are physically active have lower cancer-specific and all-cause mortality than those who are inactive (Je et al., 2013), investigating the exercise barriers among sedentary colorectal cancer patients is important. Our study found that subjects who were sedentary ( 0 MET-hour per week of physical activity) were not participating in exercise because they tend not to be physically active and they thought that they are lack of exercise skill, which showed highly statistically significance compared with their counterparts $(\mathrm{p}<0.001)$. Despite other previous studies that have found 'fatigue' as a critical barrier to exercise in patients with colorectal cancer (Courneya et al., 2005; Lynch et al., 2010), this study found that a tendency toward being sedentary and lack of confidence in doing exercise may be other important exercise barriers. Therefore, this result suggests that not only helping them alleviate their fatigue, but also educating colorectal cancer patients on the positive effects of exercise for cancer patients may be critical to encourage them to participate in exercise.

We further explored difficulties in participating in moderate to vigorous physical activity. Because ACSM have presented physical activity guidelines with a certain amount of moderate-vigorous physical activity (150 minutes of moderate to vigorous physical activity per week) to produce clinically positive effects on cancer patients (Schmitz et al., 2010), it is important to identify reasons why many of colorectal cancer patients are not participating in moderate to vigorous physical activity. In our study, only $19.7 \%$ met ACSM guidelines and $73.3 \%$ were participating in not enough amount of moderate to vigorous-intensity physical activity. Interestingly, those who were not meeting ACSM guidelines reported fear of injury, fear of adverse effects on the cancer site or treatment, and pain at site of surgery during exercise as their greater barriers to exercise as well as fatigue and poor health $(\mathrm{p}<0.001$ for all) than those who were meeting ACSM guidelines. It seems that colorectal cancer patients are reluctant to participate in higher-intensity exercise due to a fear of physical harm from exercise. Although it has been proven that participating in moderate to vigorous 
exercise is safe for cancer patients and causes physical benefits (McBride, 2010), colorectal cancer patients still appear to have misconceptions that higher-intensity exercise could harm their body or, in particular, their cancer site. However, it is true that exercise practitioners need to have special consideration for colorectal cancer patients with an ostomy in prescribing exercise (Schmitz et al., 2010). Therefore, colorectal cancer patients need be educated on safety of moderate to vigorous exercise in general, as well as practitioners in clinical field should consider colorectal cancer-specific contraindications for starting an exercise.

Differences in barriers to exercise for cancer patients found from various studies may be attributable to discrepancies in research methods. Several studies have collected exercise barrier data from cancer patients who were participating in supervised exercise intervention as part of a clinical trial, and reported that the greatest barriers to exercise are 'lack of time' or 'too busy' regardless of cancer type (Courneya et al., 2005; Ottenbacher et al., 2011). The limitation of these studies is that results from these studies could only be applied to patients who are participating in regular or scheduled exercise sessions. Meanwhile, other studies, including our current study, on exercise barriers perceived in a typical day used different questionnaires (Courneya et al., 2008; Perna et al., 2008; Rogers et al., 2008; Gho et al., 2010; Loh et al., 2011). Therefore, the results should be carefully interpreted, taking study method and assessment tools into account.

There are several limitations to this study. One of the limitations is that there is potential for ambiguity in our physical activity segment analysis, since we grouped subjects according to 'physical activity' level, but were actually exploring 'exercise' barriers. Therefore, even though exercise is regarded as a considerable part of physical activity, we should take into account subjects who did not participate in 'exercise' but did have a high physical activity level, such as those with active occupations. Also, the questionnaire used in this study was not an open-ended questionnaire. Although the questionnaire was amended for cancer patients via expert review, there could be other exercise barriers other than 21 barriers that we suggested. Lastly, the mean values for each barrier were mostly low and even the mean value of the greatest barrier was less than 3 points. We could compare exercise barriers between subgroups using the mean values; however, since all the mean values were low, the results could only be construed relatively rather than absolutely.

The importance of physical activity participation has been growing in cancer patients, and, in a practical setting, encouraging cancer patients to participate in physical activity should be more active and aggressive (Lee and Loh, 2013). To do so, understanding their constraint factors in participating in exercise is important and preceded by implementation of exercise program. In this respect, our study is significant in that we found exercise barriers for colorectal cancer patients vary according to demographic profile, treatment status, and physical activity level. Our study is the first study to identify exercise barriers among colorectal cancer patients and analyzed them according to demographics, treatment status, and physical activity level. In addition, because the exercise barriers reported in this study are barriers that patients normally perceive in a typical day, the results may be applied to general colorectal cancer patients. The findings of the current study will be applicable to practitioners who provide exercise program to colorectal cancer survivors.

\section{Acknowledgements}

This study was supported by the National Research Foundation (NRF) of Korea (no. 2010-0009048) and the National R\&D Program for Cancer Control, Ministry of Health and Welfare, Republic of Korea (no. 1120230).

\section{References}

Ahn KY, Hur H, Kim DH, et al (2013). The effects of inpatient exercise therapy on the length of hospital stay in stages I-III colon cancer patients: randomized controlled trial. Int J Colorectal Dis, 28, 643-51.

Bellizzi KM, Rowland JH, Jeffery DD, McNeel T (2005). Health behaviors of cancer survivors: examining opportunities for cancer control intervention. J Clin Oncol, 23, 8884-93.

Brawley LR, Culos-Reed SN, Angove J, Hoffman-Goetz L (2003). Understanding the barriers to physical activity for cancer patients. J Psychosocial Oncol, 20, 1-21.

Cadmus LA, Salovey P, Yu H, et al (2009). Exercise and quality of life during and after treatment for breast cancer: results of two randomized controlled trials. Psychooncology, 18, 343-52.

Caspersen CJ, Powell KE, Christenson GM (1985). Physical activity, exercise, and physical fitness: definitions and distinctions for health-related research. Public Health Rep, 100, 126-31.

Cheville AL, Kollasch J, Vandenberg J, et al (2013). A homebased exercise program to improve function, fatigue, and sleep quality in patients with Stage IV lung and colorectal cancer: a randomized controlled trial. J Pain Symptom Manage, 45, 811-21.

Chung JY, Lee DH, Park JH, et al (2013). Patterns of physical activity participation across the cancer trajectory in colorectal cancer survivors. Support Care Cancer, 21, 1605-12.

Courneya KS, Friedenreich CM, Quinney HA, et al (2003). A randomized trial of exercise and quality of life in colorectal cancer survivors. Eur J Cancer Care, 12, 347-57.

Courneya KS, Friedenreich CM, Quinney HA, et al (2005). A longitudinal study of exercise barriers in colorectal cancer survivors participating in a randomized controlled trial. Ann Behav Med, 29, 147-53.

Courneya KS, McKenzie DC, Reid RD, et al (2008). Barriers to supervised exercise training in a randomized controlled trial of breast cancer patients receiving chemotherapy. Ann Behav Med, 35, 116-22.

Gho SA, Steele JR, Munro BJ (2010). Is bra discomfort a barrier to exercise for breast cancer patients? Support Care Cancer, 18, 735-41.

Irwin ML (2009). Physical activity interventions for cancer survivors. Br J Sports Med, 43, 32-8.

Je Y, Jeon JY, Giovannucci EL, Meyerhardt JA (2013). Association between physical activity and mortality in colorectal cancer: a meta-analysis of prospective cohort studies. Int J Cancer, 133, 1905-13.

Jemal A, Bray F, Center MM, et al (2011). Global cancer statistics. CA Cancer J Clin, 61, 69-90.

Jung KW, Won YJ, Kong HJ, et al (2013). Cancer statistics in Korea: incidence, mortality, survival and prevalence in 2010. 
Cancer Res Treat, 45, 1-14.

Kruk J, Czerniak U (2013). Physical activity and its relation to cancer risk: updating the evidence. Asian Pac J Cancer Prev, 14, 3993-4003.

Leddy SK (1997). Incentives and barriers to exercise in women with a history of breast cancer. Oncol Nurs Forum, 24, 885-90.

Lee DH, Kim JY, Lee MK, et al (2013). Effects of a 12-week home-based exercise program on the level of physical activity, insulin, and cytokines in colorectal cancer survivors: a pilot study. Support Care Cancer, 21, 2537-45.

Lee JE, Loh SY (2013). Physical activity and quality of life of cancer survivors: a lack of focus for lifestyle redesign. Asian Pac J Cancer Prev, 14, 2551-5.

Loh SY, Chew SL, Lee SY (2011). Barriers to exercise: perspectives from multiethnic cancer survivors in Malaysia. Asian Pac J Cancer Prev, 12, 1483-8.

Loh SY, Lee SY, Quek KF, Murray L (2012). Barriers to participation in a randomized controlled trial of Qigong exercises amongst cancer survivors: lessons learnt. Asian Pac J Cancer Prev, 13, 6337-42.

Lynch BM, Owen N, Hawkes AL, Aitken JF (2010). Perceived barriers to physical activity for colorectal cancer survivors. Support Care Cancer, 18, 729-34.

Maryam A, Fazlollah A, Eesa M, Ebrahim H, Abbas VF (2010). The effect of designed exercise programme on quality of life in women with breast cancer receiving chemotherapy. Scand J Caring Sci, 24, 251-8.

McBride D (2010). ACSM releases new guidelines for physical activity for patients with cancer. ONS Connect, 25, 16.

Nelson JP (1991). Perceived health, self-esteem, health habits, and perceived benefits and barriers to exercise in women who have and who have not experienced stage I breast cancer. Oncol Nurs Forum, 18, 1191-7.

Ottenbacher AJ, Day RS, Taylor WC, et al (2011). Exercise among breast and prostate cancer survivors--what are their barriers? J Cancer Surviv, 5, 413-9.

Ottenbacher AJ, Sloane R, Snyder DC, et al (2013). Cancerspecific concerns and physical activity among recently diagnosed breast and prostate cancer survivors. Integr Cancer Ther, 12, 206-12.

Peddle CJ, Au HJ, Courneya KS (2008). Associations between exercise, quality of life, and fatigue in colorectal cancer survivors. Dis Colon Rectum, 51, 1242-8.

Perna FM, Craft L, Carver CS, Antoni MH (2008). Negative affect and barriers to exercise among early stage breast cancer patients. Health Psychol, 27, 275-9.

Rogers LQ, Courneya KS, Robbins KT, et al (2008). Physical activity correlates and barriers in head and neck cancer patients. Support Care Cancer, 16, 19-27.

Rogers LQ, Courneya KS, Shah P, Dunnington G, Hopkins-Price $P$ (2007). Exercise stage of change, barriers, expectations, values and preferences among breast cancer patients during treatment: a pilot study. Eur J Cancer Care, 16, 55-66.

Rogers LQ, Courneya KS, Verhulst S, et al (2006). Exercise barrier and task self-efficacy in breast cancer patients during treatment. Support Care Cancer, 14, 84-90.

Schmitz KH, Courneya KS, Matthews C, et al (2010). American College of Sports Medicine roundtable on exercise guidelines for cancer survivors. Med Sci Sports Exerc, 42, 1409-26.

Seong CH, Yoo LK, Jang CH (2008). Conceptual structure of exercise constraints and the difference according to the stage of change in the aged. Korean J Sport Psychology, 19, 115-33.

Servaes P, Verhagen C, Bleijenberg G (2002). Fatigue in cancer patients during and after treatment: prevalence, correlates and interventions. Eur J Cancer, 38, 27-43.
DOI:http://dx.doi.org/10.7314/APJCP.2014.15.18.7539

Exercise Barriers in Korean Colorectal Cancer Patients

Shariati A, Haghighi S, Fayyazi S, Tabesh H, Kalboland MM (2010). The effect of exercise on the severity of the fatigue in colorectal cancer patients who received chemotherapy in Ahwaz. Iran J Nurs Midwifery Res, 15, 145-9.

Sherman KA, Heard G, Cavanagh KL (2010). Psychological effects and mediators of a group multi-component program for breast cancer survivors. J Behav Med, 33, 378-91.

Siegel R, Naishadham D, Jemal A (2013). Cancer statistics, 2013. CA Cancer J Clin, 63, 11-30.

Spazzapan S, Bearz A, Tirelli U (2004). Fatigue in cancer patients receiving chemotherapy: an analysis of published studies. Ann Oncol, 15, 1576. 